This item was submitted to Loughborough's Research Repository by the author.

Items in Figshare are protected by copyright, with all rights reserved, unless otherwise indicated.

\title{
Cone extensions of polyhedral production technologies
}

PLEASE CITE THE PUBLISHED VERSION

https://doi.org/10.1016/j.ejor.2019.01.031

\section{PUBLISHER}

(c) Elsevier

\section{VERSION}

AM (Accepted Manuscript)

\section{PUBLISHER STATEMENT}

This paper was accepted for publication in the journal European Journal of Operational Research and the definitive published version is available at https://doi.org/10.1016/j.ejor.2019.01.031

\section{LICENCE}

CC BY-NC-ND 4.0

\section{REPOSITORY RECORD}

Podinovski, Victor, and Tatiana Bouzdine-Chameeva. 2019. "Cone Extensions of Polyhedral Production Technologies”. Loughborough University. https://hdl.handle.net/2134/36649. 


\title{
Cone extensions of polyhedral production technologies
}

\author{
Victor V. Podinovski ${ }^{\mathrm{a}, *}$, Tatiana Bouzdine-Chameeva ${ }^{\mathrm{b}}$ \\ ${ }^{a}$ School of Business and Economics, Loughborough University, Loughborough LE11 3TU, UK \\ ${ }^{b}$ KEDGE Business School, 680 cours de la Libération, 33405 Talence Cedex, France
}

\begin{abstract}
In data envelopment analysis, cone extensions of production technologies are often used for the estimation of scale efficiency of decision making units. Furthermore, the non-increasing and non-decreasing returns-to-scale (NIRS and NDRS) technologies are often used for their returns-to-scale characterization. Although a number of new production technologies have recently been developed in the literature, their cone, NIRS and NDRS extensions have not always been fully explored. In this paper, we obtain general results that show how these extensions can be obtained, for an arbitrary polyhedral technology. We illustrate the usefulness of our results by examples.
\end{abstract}

Keywords: Data envelopment analysis, Cone technology, Scale efficiency, Returns to scale, Network data envelopment analysis

\section{Introduction}

Most convex production technologies developed in the literature on data envelopment analysis (DEA) are polyhedral sets. Podinovski, Chambers, Atici, and Deineko (2016) refer to such technologies as polyhedral technologies. Examples include the standard constant and variable returns-to-scale (CRS and VRS) technologies of Charnes, Cooper and Rhodes (1978) and Banker, Charnes and Cooper (1984), their extensions by production trade-offs (Podinovski, 2004d; Podinovski \& Bouzdine-Chameeva, 2013), the hybrid returns-to-scale technology (Podinovski, 2004c), different technologies with multiple component processes (Cherchye, De Rock, Dierynck, Roodhooft, \& Sabbe, 2013; Podinovski, Olesen, \& Sarrico, 2018), various network technologies (Färe, Grosskopf, \& Whittaker, 2007; Kao, 2017), and technologies incorporating the assumptions of weak disposability of outputs (Kuosmanen, 2005) and inputs (Mehdiloozad \& Podinovski, 2018).

A common challenge in developing new technologies is the need to explain how the established concepts and approaches commonly used with the existing technologies could be applied to the new proposed technologies. An example of this is the notion of scale efficiency. In DEA, this concept was defined by Banker et al. (1984) and Banker (1984).

According to Banker (1984), testing for scale efficiency of a decision making unit (DMU) in the VRS technology requires evaluating its efficiency in the VRS technology and in the reference cone technology, which is the CRS technology of Charnes et al. (1978). A related question of returns-to-scale (RTS) characterization is often addressed by using either a nonincreasing or non-decreasing (NIRS and NDRS) reference technologies (Färe, Grosskopf, \& Lovell, 1983, 1985; Kerstens and Vanden Eeckaut, 1999; Podinovski, 2004a, 2004b).

\footnotetext{
*Corresponding author

Email addresses: v.podinovski@lboro.ac.uk (Victor V. Podinovski), tatiana.chameeva@kedgebs.com (Tatiana Bouzdine-Chameeva)
} 
The above common approaches to the evaluation of scale efficiency and RTS conceptually extend to any other polyhedral technology. ${ }^{1}$ However, implementing these approaches assumes that we know how to construct the required cone, NIRS and NDRS reference technologies for the given polyhedral technology. This task is not always simple, and for a number of recently introduced polyhedral technologies, it has remained unexplored or has been approached heuristically, using an unverified analogy with the VRS technology.

In this paper, we consider the whole class of polyhedral technologies. All such technologies are closed convex sets which can be stated in a particular form discussed in Section 2. We obtain general results that show how the cone, NIRS and NDRS reference technologies can be obtained for any polyhedral technology. These results make bespoke, and often lengthy, rigorous developments of such technologies in all special cases largely unnecessary. We illustrate our approach by several examples in which the statements of the reference technologies may not be immediately obvious but are easily obtained by using our general results.

We proceed as follows. In Section 2, we briefly introduce the general statement of a polyhedral technology. In Section 3, we establish several mathematical results clarifying the role of the reference cone technology in the evaluation of scale efficiency. In Section 4, we prove our main result that shows how the reference cone technology is constructed in the general case. In Section 5, we discuss the construction of the NIRS and NDRS technologies. In Section 6, we illustrate our results by examples. In Section 7, we consider the construction of the cone technology from the dual perspective. A summary of our results is presented in Section 8. All mathematical proofs are given in Appendix A.

\section{Polyhedral technologies}

Let $\mathcal{T} \in \mathbb{R}_{+}^{m+s}$ be a polyhedral technology with $m$ inputs and $s$ outputs. ${ }^{2}$ DMUs are denoted $(x, y)$, where $x \in \mathbb{R}_{+}^{m}$ is the vector of inputs and $y \in \mathbb{R}_{+}^{s}$ is the vector of outputs.

As follows from Podinovski et al. (2016), any polyhedral technology $\mathcal{T}$ can be stated as the set of all DMUs $(x, y) \in \mathbb{R}_{+}^{m+s}$ for which there exists a vector $z$ of some dimension $q$ such that the following conditions (taking on a more specific form for particular technologies) are true:

$$
\begin{aligned}
& A z=x, \\
& B z=y, \\
& C z=d, \\
& z \geq 0 .
\end{aligned}
$$

In statement (1), $A, B$ and $C$ are some matrices of dimensions $m \times q, s \times q$ and $p \times q$, respectively. The vector $d$ is a constant vector of dimension $p$.

\footnotetext{
${ }^{1}$ For nonconvex technologies, which excludes polyhedral technologies, the reference technology approaches identify global types of RTS (Podinovski, 2004a, 2004b) which are different from the conventional local types of RTS based on the notion of scale elasticity. For convex, including polyhedral technologies, the local and global RTS characterizations are the same (Podinovski, 2017). A common example of nonconvex technologies is the free disposal hull (FDH) of Deprins, Simar, and Tulkens (1984). For the FDH technology, special enumeration algorithms for the evaluation of global RTS have been developed and shown to be computationally superior to the generic approaches based on the reference technologies - see, e.g, Soleimanidamaneh, Jahanshahloo, and Reshadi (2006) and Cesaroni and Giovannola (2015).

${ }^{2}$ In a finite-dimensional space, a polyhedral set is defined as the intersection of a finite number of closed half-spaces (Rockafellar, 1970).
} 
The parameter conditions (1c) are optional and may not be specified for some technologies. An individual equality $t=1, \ldots, p$ in the parameter conditions (1c) is homogeneous if the constant on its right-hand side is equal to zero, i.e., $d_{t}=0$. Otherwise, i.e., if $d_{t} \neq 0$, the corresponding parameter condition $t$ is nonhomogeneous.

Most polyhedral technologies assume free disposability of all inputs and outputs. For such technologies, it is common to state the input and output conditions (1a) and (1b) as inequalities. Alternatively, the same conditions can be represented as equalities, by introducing a slack variable for each inequality. The above general statement (1) of technology $\mathcal{T}$ assumes the latter approach and treats any slack variables as components of vector $z$.

For example, consider the VRS technology of Banker et al. (1984) for which conditions (1) take on a more specific form:

$$
\begin{aligned}
& X \lambda+s_{x}=x, \\
& Y \lambda-s_{y}=y, \\
& 1^{\top} \lambda=1, \\
& \lambda, s_{x}, s_{y} \geq 0 .
\end{aligned}
$$

In the above statement, $X$ and $Y$ are the $m \times n$ and $s \times n$ matrices whose columns are the input and output vectors of the observed DMUs $\left(x_{j}, y_{j}\right), j=1, \ldots, n$, respectively.

It is clear that statement (2) is a special case of (1). In particular, the vector $z$ in (1) is the combined vector $\left(\lambda, s_{x}, s_{y}\right)$ in (2) whose dimension is $q=n+m+s$. The matrix $A$ in (1a) is the combined matrix $\left[X, I_{m \times m}, 0_{m \times s}\right]$ in (2a), where $I_{m \times m}$ and $0_{m \times s}$ are the unit (identity) and null (zero) matrices of the specified dimensions. Similarly, the matrix $B$ in (1b) is the combined matrix $\left[Y, 0_{s \times m},-I_{s \times s}\right]$ in $(2 \mathrm{~b})$. Finally, the matrix $C$ in (1c) has a single row in conditions (2c) stated as the combined vector $\left(1_{n}, 0_{m}, 0_{s}\right)$ whose first $n$ components are each equal to 1 and the remaining $m+s$ components are zeros. The vector $d$ in (1c) corresponds to the scalar 1 in $(2 \mathrm{c})$. Note that the parameter condition (2c) consists of a single equality and is nonhomogeneous.

For many other technologies, conditions (1) have a more complex structure. We consider examples of such technologies in Section 6.

\section{The role of cone technologies}

We start with the following definition.

Definition 1. Technology $\mathcal{T}$ is a cone technology if, for any $(x, y) \in \mathcal{T}$ and any $\alpha \geq 0$, $(\alpha x, \alpha y) \in \mathcal{T}$.

Let $\mathcal{T}$ be a polyhedral technology stated by conditions (1). It is clear that, if all parameter conditions (1c) are homogeneous (or are not specified, as for the CRS model), the polyhedral technology $\mathcal{T}$ defined by $(1)$ is a cone technology. ${ }^{3}$

Cone technologies play an important role in efficiency analysis. For example, the notions of scale efficiency and most productive scale size (MPSS) introduced for the VRS technology by Banker (1984) typically involve the evaluation of the output radial efficiency of the $\mathrm{DMU}_{o}=\left(x_{o}, y_{o}\right)$ of interest in the reference CRS technology. The latter is the cone technology generated by VRS technology. Below we show that a similar approach is applicable to

\footnotetext{
${ }^{3}$ Various properties of convex cones have been extensively studied in the literature (Rockafellar, 1970; Rockafellar \& Wets, 1998).
} 
any other polyhedral technology $\mathcal{T}$. Discussing this requires some additional mathematical development.

Following Banker (1984), DMU $\mathrm{DM}_{o}$ is at MPSS if, for all DMUs $\left(\alpha x_{o}, \beta y_{o}\right) \in \mathcal{T}$, where $\alpha>0$, we have $\beta / \alpha \leq 1$. In other words, $\mathrm{DMU}_{o}$ is at MPSS if the supremum of the following program is attained at $\alpha=\beta=1$ and is, therefore, equal to 1 :

$$
\begin{aligned}
\sup & \beta / \alpha \\
\text { subject to } & \left(\alpha x_{o}, \beta y_{o}\right) \in \mathcal{T}, \\
& \alpha, \beta>0 .
\end{aligned}
$$

It is clear that, if $\mathrm{DMU}_{o}$ is at MPSS, it is output radial efficient in $\mathcal{T}$ but the converse is not necessarily true. In order to linearize program (3), introduce the reference cone technology $\mathcal{C}$ as follows:

$$
\mathcal{C}=\left\{(x, y) \in \mathbb{R}^{m+s} \mid \exists(\tilde{x}, \tilde{y}) \in \mathcal{T}, \delta \geq 0:(x, y)=(\delta \tilde{x}, \delta \tilde{y})\right\} .
$$

Technology $\mathcal{C}$ includes all rays in $\mathbb{R}_{+}^{m+s}$ starting from the origin and passing through the points (DMUs) in technology $\mathcal{T}$. It is straightforward to prove that technology $\mathcal{C}$ coincides with the intersection of all cone technologies $\mathcal{T}^{\prime}$ such that $\mathcal{T} \subseteq \mathcal{T}^{\prime}$. Therefore, $\mathcal{C}$ is the smallest cone technology generated by $\mathcal{T}$.

Proposition 1. The supremum of program (3) is equal to the supremum of the program

$$
\begin{aligned}
\sup & \beta \\
\text { subject to } & \left(x_{o}, \beta y_{o}\right) \in \mathcal{C} .
\end{aligned}
$$

If the supremum in either program (3) or (5) is attained, then it is attained in both programs.

Note that, because $\left(x_{o}, y_{o}\right) \in \mathcal{C}$, the condition $\beta>0$ in program (5) is redundant and is omitted. A similar observation applies to programs (7), (8) and (9) presented below.

We now address the question of attainability of the suprema in programs (3) and (5). If $\mathcal{T}$ is the conventional VRS technology, $\mathcal{C}$ is the conventional CRS technology, which is a closed cone. In this case, the supremum in (5) is always attained and is the inverse of the output radial efficiency of $\mathrm{DMU}_{o}$ in the CRS technology. By Proposition 1, the supremum in (3) is also attained. Therefore, if $\mathcal{T}$ is the VRS technology, we can substitute the supremum of the objective functions in (3) and (5) by their maximum, and the latter program becomes a linear program.

In contrast, for an arbitrary polyhedral technology $\mathcal{T}$, the corresponding cone technology $\mathcal{C}$ may not be a closed set and the suprema in programs (3) and (5) may not be attained. This possibility was illustrated by an example in Podinovski (2017). In this case, the problem that we face in solving program $(5)$ is that $\mathcal{C}$ is not a polyhedral technology (as any polyhedral technology is a closed set) which cannot therefore be conveniently represented by linear conditions (1). To overcome this problem and find an operational way of solving program (5), consider the closure $\overline{\mathcal{C}}$ of technology $\mathcal{C}$ :

$$
\overline{\mathcal{C}}=\operatorname{cl} \mathcal{C} .
$$

Technology $\overline{\mathcal{C}}$ is the intersection of all closed cone technologies $\overline{\mathcal{T}}$ such that $\mathcal{T} \subseteq \overline{\mathcal{T}}$. In this sense, $\overline{\mathcal{C}}$ is the smallest closed cone technology generated by $\mathcal{T}$.

We now prove that the supremum of $\beta$ in program (5) representing the inverse of the output radial efficiency of DMU $\left(x_{o}, y_{o}\right)$ in the generally not closed technology $\mathcal{C}$ does not 
change if we replace it by its closure $\overline{\mathcal{C}}$. Below we establish a more general result from which the required statement follows. Namely, we consider the assessment of efficiency of $\operatorname{DMU}\left(x_{o}, y_{o}\right)$ in the direction of an arbitrary vector $g=\left(g_{x}, g_{y}\right) \in \mathbb{R}_{+}^{m+s}$, such that $g \neq 0$ (Chambers, Chung, \& Färe, 1998). Consider the program:

$$
\begin{aligned}
\sup & \beta \\
\text { subject to } & \left(x_{o}-\beta g_{x}, y_{o}+\beta g_{y}\right) \in \mathcal{C},
\end{aligned}
$$

and the program obtained from it by replacing technology $\mathcal{C}$ by $\overline{\mathcal{C}}$ :

$$
\begin{aligned}
\sup & \beta \\
\text { subject to } & \left(x_{o}-\beta g_{x}, y_{o}+\beta g_{y}\right) \in \overline{\mathcal{C}},
\end{aligned}
$$

The following result is not trivial, and its proof relies on the explicit form of the technology $\overline{\mathcal{C}}$ obtained in the next section. ${ }^{4}$

Proposition 2. The suprema of $\beta$ in programs (7) and (8) are equal.

Because program (5) is a special case of $(7),{ }^{5}$ we have

Corollary 1. The supremum of program (5) does not change if we replace the cone technology $\mathcal{C}$ in its constraints by its closure $\overline{\mathcal{C}}{ }^{6}$

In the next section we show that, for any polyhedral technology $\mathcal{T}$, its closed cone extension $\overline{\mathcal{C}}$ is a polyhedral technology whose statement is obtained by a simple modification of the statement (1) of technology $\mathcal{T}$. This means that evaluating MPSS in an arbitrary polyhedral technology $\mathcal{T}$ is a straightforward task that requires solving the following linear program: ${ }^{7}$

$$
\begin{aligned}
\max & \beta \\
\text { subject to } & \left(x_{o}, \beta y_{o}\right) \in \overline{\mathcal{C}} .
\end{aligned}
$$

Using all the results obtained above, we conclude that $\mathrm{DMU}_{o}$ in an arbitrary polyhedral technology is at MPSS if and only if it is output radial efficient in the closed cone technology $\overline{\mathcal{C}}$ generated by the original polyhedral technology $\mathcal{T}$.

Furthermore, following Banker et al. (1984), the output radial efficiency of $\mathrm{DMU}_{o}$ in the closed convex technology $\overline{\mathcal{C}}$, i.e., the inverse of the optimal value of program (9), is interpretable as the aggregate technical and scale efficiency of $\mathrm{DMU}_{o}$. The scale efficiency of $\mathrm{DMU}_{o}$ is found as the ratio of its output radial efficiency in technology $\overline{\mathcal{C}}$ to its output radial efficiency (technical efficiency) in the original polyhedral technology $\mathcal{T}$.

\footnotetext{
${ }^{4}$ This result is not valid if we substitute $\mathcal{C}$ and $\overline{\mathcal{C}}$ in programs $(7)$ and (8) by, respectively, an arbitrary convex set and its closure. An example of this was given in Podinovski (2017).

${ }^{5}$ Program (7) becomes (5) if we take $g_{x}=0, g_{y}=y_{o}$ and redefine $1+\beta$ in the former program as $\beta$ in the latter.

${ }^{6}$ This does not imply that the attainability of the supremum is unaffected. The resulting program (9) is a linear program. Its maximum may be attained even if the supremum in (5) is not.

${ }^{7}$ In line with the conventional notation used in linear programming, we replace the supremum by maximum of the objective function. If the objective function $\beta$ of the linear program (9) is bounded above, then its maximum is attained. However, without additional assumptions, for an arbitrary technology $\overline{\mathcal{C}}$ this cannot be guaranteed. An example of this was explored by Podinovski and Bouzdine-Chameeva (2013) who showed that, in the case of the VRS or CRS technology expanded by weight restrictions, program (9) may have an unbounded optimal value, which indicates a particular error in the specification of the technology.
} 


\section{The closed cone technology}

Below we prove our main result. It establishes that the closed cone technology $\overline{\mathcal{C}}$ generated by any polyhedral technology $\mathcal{T}$ is obtained by a simple modification of the general statement of the latter technology. We illustrate the usefulness of this result by the examples in Section 6.

Proposition 3. Technology $\overline{\mathcal{C}}$ is a polyhedral technology. It is the set of all DMUs $(x, y) \in$ $\mathbb{R}_{+}^{m+s}$ for which there exist $z \in \mathbb{R}^{q}$ and $\alpha \in \mathbb{R}$ such that the following conditions are true:

$$
\begin{aligned}
& A z=x \\
& B z=y \\
& C z=\alpha d \\
& z, \alpha \geq 0
\end{aligned}
$$

By Proposition 3, we can restate program (3) in the explicit linear programming form:

$$
\begin{aligned}
\max & \beta \\
\text { subject to } & A z=x_{o}, \\
& B z=\beta y_{o} \\
& C z=\alpha d, \\
& z, \alpha \geq 0, \beta \text { sign free. }
\end{aligned}
$$

Corollary 2. If the parameter conditions (1c) include a single nonhomogeneous condition (and any other parameter conditions are homogeneous), then technology $\overline{\mathcal{C}}$ can alternatively be stated in the form (1) from which this nonhomogeneous condition is removed.

As an example, consider the VRS technology of Banker et al. (1984). The statement (2) of this technology contains a single nonhomogeneous equality (2c). By Corollary 2, the technology $\overline{\mathcal{C}}$ is obtained by removing this condition. The remaining conditions define the CRS technology of Charnes et al. (1978) which is the closed cone technology generated by the VRS technology.

\section{The NIRS and NDRS technologies}

The NIRS and NDRS technologies generated by technology $\mathcal{T}$ are used in the method of testing RTS developed by Färe et al. (1983) and in its variants explored by Kerstens and Vanden Eeckaut (1999), Briec, Kerstens, Leleu and Vanden Eeckaut (2000), Podinovski (2004a, 2004b, 2017), and Cesaroni, Kerstens, and Van de Woestyne (2017). Below we show that, if technology $\mathcal{T}$ is a polyhedral technology, its reference NIRS and NDRS technologies can be obtained by a simple modification of its statement (1).

We start by giving the following definitions.

Definition 2. $\mathcal{T}$ is a NIRS technology if, for any $(x, y) \in \mathcal{T}$ and any $\alpha \in[0,1],(\alpha x, \alpha y) \in \mathcal{T}$.

Definition 3. $\mathcal{T}$ is a NDRS technology if, for any $(x, y) \in \mathcal{T}$ and any $\alpha \geq 1,(\alpha x, \alpha y) \in \mathcal{T}$.

The NIRS technology $\mathcal{H}$ and the NDRS technology $\mathcal{G}$ generated by technology $\mathcal{T}$ are conventionally defined as follows:

$$
\mathcal{H}=\left\{(x, y) \in \mathbb{R}^{m+s} \mid \exists(\tilde{x}, \tilde{y}) \in \mathcal{T}, \delta \in[0,1]:(x, y)=(\delta \tilde{x}, \delta \tilde{y})\right\},
$$




$$
\mathcal{G}=\left\{(x, y) \in \mathbb{R}^{m+s} \mid \exists(\tilde{x}, \tilde{y}) \in \mathcal{T}, \delta \geq 0:(x, y)=(\delta \tilde{x}, \delta \tilde{y})\right\} .
$$

It is straightforward to prove that $\mathcal{H}$ and $\mathcal{G}$ coincide with the intersection of all NIRS and, respectively, NDRS technologies $\mathcal{T}^{\prime}$ such that $\mathcal{T} \subseteq \mathcal{T}^{\prime}$. Therefore, $\mathcal{H}$ and $\mathcal{G}$ are, respectively, the smallest NIRS and NDRS technologies generated by $\mathcal{T}$.

It is known that, if $\mathcal{T}$ is the conventional VRS technology of Banker et al. (1984), the corresponding NIRS and NDRS technologies are both closed sets. For an arbitrary polyhedral technology $\mathcal{T}$, the corresponding NDRS technology $\mathcal{G}$ is always a closed set but the NIRS technology $\mathcal{H}$ may be not closed (Podinovski, 2017).

Proposition 4. The NDRS technology $\mathcal{G}$ is a closed set.

In the cases in which the NIRS technology $\mathcal{H}$ is not a closed set, it is not a polyhedral technology (because any polyhedral technology is closed). In such cases, $\mathcal{H}$ cannot be stated in the form (1). Let $\overline{\mathcal{H}}$ be the closure of $\mathcal{H}$ :

$$
\overline{\mathcal{H}}=\operatorname{cl} \mathcal{H} .
$$

Proposition 5. Technology $\overline{\mathcal{H}}$ is stated by conditions in (10) with the additional condition $\alpha \leq 1$. Technology $\mathcal{G}$ is stated by conditions in (10) with the additional condition $\alpha \geq 1$.

Similar to Proposition 2, it can be proved that the efficiency of any $\mathrm{DMU}_{o}$ evaluated in the direction of any vector $g \neq 0$, of which the input and output radial efficiency of $\mathrm{DMU}_{o}$ is a special case, is the same in technology $\mathcal{H}$ and its closure $\overline{\mathcal{H}}$.

According to Proposition 5, assessing the output radial efficiency of $\mathrm{DMU}_{o}$ in the closed NIRS and NDRS technologies $\overline{\mathcal{H}}$ and $\mathcal{G}$ requires solving the linear program (11) with the additional constraints $\alpha \leq 1$ and $\alpha \geq 1$, respectively.

\section{Examples}

Below we consider examples of different polyhedral technologies. We show how their closed cone, NIRS and NDRS extensions can be constructed using Propositions 3 and 5.

\subsection{A two-stage network technology}

Consider the following two-stage network production technology (Kao, 2014, 2017; Liang, Cook, \& Zhu, 2008; Sahoo, Zhu, Tone, \& Klemen, 2014). The first stage uses the vector of inputs $x \in \mathbb{R}_{+}^{m}$ to produce the vector of intermediate outputs $w \in \mathbb{R}_{+}^{l}$. The latter vector is used as the input vector of the second stage which produces the vector of final outputs $y \in \mathbb{R}_{+}^{s}$.

Let observed DMUs be the triplets $\left(x_{j}, w_{j}, y_{j}\right), j=1, \ldots, n$. Denote $X, W$ and $Y$ the $m \times n, l \times n$ and $s \times n$ matrices whose columns are the vectors $x_{j}, w_{j}$ and $y_{j}, j=1, \ldots, n$, respectively. Following Liang et al. (2008) and Sahoo et al. (2014), consider the VRS network technology $\mathcal{T}_{\mathrm{N}}$ generated by the above observed DMUs and stated in the input and final output dimensions only. To show that this technology is a special case of the general statement of polyhedral technology (1), we state it as follows.

Technology $\mathcal{T}_{\mathrm{N}}$ is the set of all DMUs $(x, y) \in \mathbb{R}_{+}^{m+s}$ for which there exist vectors $\lambda, \mu \in$ $\mathbb{R}^{n}$, the vector of intermediate outputs $w \in \mathbb{R}^{l}$, and slack vectors $s_{x}, s_{y}, s_{w}^{1}$ and $s_{w}^{2}$ of 
appropriate dimensions such that

$$
\begin{aligned}
& X \lambda+s_{x}=x, \\
& Y \mu-s_{y}=y, \\
& W \lambda-w-s_{w}^{1}=0, \\
& W \mu-w+s_{w}^{2}=0, \\
& 1^{\top} \lambda=1, \\
& 1^{\top} \mu=1, \\
& \lambda, \mu, w, s_{x}, s_{y}, s_{w}^{1}, s_{w}^{2} \geq 0 .
\end{aligned}
$$

We are now concerned with the following question: what is the closed cone extension $\overline{\mathcal{C}}_{\mathrm{N}}$ generated by technology $\mathcal{T}_{\mathrm{N}}$ ? To answer this question, note that (12) is a special case of the general statement of polyhedral technology (1). In particular, we can view $z$ as the combined vector $\left(\lambda, \mu, w, s_{x}, s_{y}, s_{w}^{1}, s_{w}^{2}\right)$. Equalities (12a) and (12b) correspond to (1a) and (1b). The homogeneous conditions (12c) and (12d) and the two nonhomogeneous scalar conditions (12e) and (12f) are the parameter conditions (1c).

By Proposition 3, the closed cone technology $\overline{\mathcal{C}}_{\mathrm{N}}$ is defined by conditions (12) in which we introduce an additional scalar variable $\alpha$ and replace equalities (12e) and (12f) by the following conditions:

$$
\begin{aligned}
& 1^{\top} \lambda=\alpha, \\
& 1^{\top} \mu=\alpha, \\
& \alpha \geq 0 .
\end{aligned}
$$

By Proposition 5, the NIRS and NDRS technologies $\overline{\mathcal{H}}_{\mathrm{N}}$ and $\mathcal{G}_{\mathrm{N}}$ are stated by incorporating the further condition $\alpha \leq 1$ or $\alpha \geq 1$, respectively.

Note that, if we simply omit the two equalities (12e) and (12f), we also obtain a closed cone technology $\mathcal{T}_{\mathrm{N}}^{\prime}$ that extends $\mathcal{T}_{\mathrm{N}}$. However, this would be equivalent to replacing (12e) and (12f) by the conditions

$$
\begin{aligned}
& 1^{\top} \lambda=\alpha_{1}, \\
& 1^{\top} \mu=\alpha_{2}, \\
& \alpha_{1}, \alpha_{2} \geq 0 .
\end{aligned}
$$

It is easy to show that technology $\mathcal{T}_{\mathrm{N}}^{\prime}$ is generally larger than $\overline{\mathcal{C}}_{\mathrm{N}}$ and is therefore not suitable for the evaluation of MPSS and scale efficiency.

Similarly, replacing both equalities (12e) and (12f) by the " $\leq$ " or " $\geq$ " inequalities is incorrect and produces the technologies that are larger than the NIRS and NDRS technologies $\overline{\mathcal{H}}_{\mathrm{N}}$ and $\mathcal{G}_{\mathrm{N}}$, respectively.

\subsection{Technologies with bounded measures}

Consider the bounded CRS technology $\mathcal{T}_{\mathrm{B}}$ introduced by Cooper, Pastor, Borras, Aparicio, and Pastor (2011). ${ }^{8}$ Let $x \in \mathbb{R}_{+}^{m}$ be the vector of inputs and $y \in \mathbb{R}_{+}^{s}$ the vector of outputs. Denote observed DMUs $\left(x_{j}, y_{j}\right), j=1, \ldots, n$. Denote $X$ and $Y$ the $m \times n$ and $s \times n$ matrices whose columns are the vectors $x_{j}$ and $y_{j}, j=1, \ldots, n$, respectively.

\footnotetext{
${ }^{8}$ Further extensions of technology $\mathcal{T}_{\mathrm{B}}$ were developed by Pastor, Aparicio, Alcaraz, Vidal, and Pastor (2015).
} 
It is additionally assumed that there exist a lower bound on each input and an upper bound on each output, represented by the vectors $\underline{x} \in \mathbb{R}_{+}^{m}$ and $\bar{y} \in \mathbb{R}_{+}^{n}$, respectively.

As shown by Cooper et al. (2011), technology $\mathcal{T}_{\mathrm{B}}$ is the set of all DMUs $(x, y) \in \mathbb{R}_{+}^{m+s}$ for which there exist a vector $\lambda \in \mathbb{R}^{n}$ and slack vectors $s_{x}, s_{y}, s_{x}^{\prime}$ and $s_{y}^{\prime}$ of appropriate dimensions such that

$$
\begin{aligned}
& X \lambda+s_{x}=x, \\
& Y \lambda-s_{y}=y, \\
& X \lambda-s_{x}^{\prime}=\underline{x} \\
& Y \lambda+s_{y}^{\prime}=\bar{y} \\
& \lambda, s_{x}, s_{y}, s_{x}^{\prime}, s_{y}^{\prime} \geq 0 .
\end{aligned}
$$

Although the conventional CRS technology is a closed cone technology, its bounded analogue $\mathcal{T}_{\mathrm{B}}$ is not. A question therefore arises as to what is the closed cone technology $\overline{\mathcal{C}}_{\mathrm{B}}$ generated by technology $\mathcal{T}_{\mathrm{B}}$ ?

Note that (14) is a special case of the general statement of polyhedral technology (1). In particular, vector $z$ in (1) is the combined vector $\left(\lambda, s_{x}, s_{y}, s_{x}^{\prime}, s_{y}^{\prime}\right)$ in (14). Conditions (14a) and (14b) correspond to conditions (1a) and (1b). The nonhomogeneous conditions (14c) and $(14 \mathrm{~d})$ are parameter conditions (1c).

By Proposition 3, the statement of technology $\overline{\mathcal{C}}_{\mathrm{B}}$ is obtained from (14) by introducing an additional scalar variable $\alpha$ and replacing its constraints (14c) and (14d) by the following:

$$
\begin{aligned}
& X \lambda-s_{x}^{\prime}=\alpha \underline{x}, \\
& Y \lambda+s_{y}^{\prime}=\alpha \bar{y}, \\
& \alpha \geq 0 .
\end{aligned}
$$

By Proposition 5, the statements of the NIRS and NDRS technologies $\overline{\mathcal{H}}_{\mathrm{B}}$ and $\mathcal{G}_{\mathrm{B}}$ require the incorporation of the additional inequality $\alpha \leq 1$ and $\alpha \geq 1$, respectively.

\subsection{Technologies with weight restrictions}

Many applications of DEA involve the specification of weight restrictions in the multiplier models based on the conventional VRS or CRS production technologies (Allen, Athanassopoulos, Dyson, \& Thanassoulis, 1997; Dyson and Thanassoulis, 1988; Podinovski, 2015). Below we consider the VRS models with weight restrictions.

Using notation from the previous example, let $X$ and $Y$ be the $m \times n$ and $s \times n$ matrices whose columns are the input and output vectors of the observed DMUs $\left(x_{j}, y_{j}\right), j=1, \ldots, n$, respectively. Let $u \in \mathbb{R}_{+}^{s}$ and $v \in \mathbb{R}_{+}^{m}$ be the vectors of the output and input weights used in either the input or output-oriented multiplier VRS model. The most common homogeneous weight restrictions are stated in the form:

$$
Q_{t}^{\top} u-P_{t}^{\top} v \leq 0, \quad t=1, \ldots, K,
$$

where $Q_{t} \in \mathbb{R}^{s}$ and $P_{t} \in \mathbb{R}^{m}$ are constant vectors whose components can be positive, negative or equal to zero. Define the matrices $Q$ and $P$ whose dimensions are $s \times K$ and $m \times K$, respectively, and whose columns are vectors $Q_{t}$ and $P_{t}, t=1, \ldots, K$.

The incorporation of weight restrictions (16) in the multiplier VRS model leads to the corresponding change in the dual envelopment model. As shown by Podinovski (2004d), the dual terms to weight restrictions (16) are interpretable as production trade-offs. Furthermore, the evaluation of the input or output radial efficiency in then performed in the expanded technology denoted $\mathcal{T}_{\text {TO }}$. 
Technology $\mathcal{T}_{\text {TO }}$ is stated as the set of all DMUs $(x, y) \in \mathbb{R}_{+}^{m+s}$ for which there exist vectors $\lambda \in \mathbb{R}^{n}, \pi \in \mathbb{R}^{K}$ and slack vectors $s_{x}$ and $s_{y}$ of appropriate dimensions such that

$$
\begin{aligned}
& X \lambda+P \pi+s_{x}=x, \\
& Y \lambda+Q \pi-s_{y}=y, \\
& 1^{\top} \lambda=1, \\
& \lambda, \pi, s_{x}, s_{y} \geq 0 .
\end{aligned}
$$

It is clear that the above statement of technology $\mathcal{T}_{\text {TO }}$ is a special case of the general statement (1) if we define $z$ as the combined vector $\left(\lambda, \pi, s_{x}, s_{y}\right)$. Then conditions (17a), $(17 \mathrm{~b})$ and $(17 \mathrm{c})$ correspond to (1a), (1b) and (1c), respectively.

By Corollary 2 , the closed cone extension $\overline{\mathcal{C}}_{\mathrm{TO}}$ of technology $\mathcal{T}_{\mathrm{TO}}$ is obtained by the statement (17) from which we need to remove equality $(17 \mathrm{c})$. Therefore, technology $\overline{\mathcal{C}}_{\mathrm{TO}}$ is the conventional CRS technology expanded by the trade-offs dual to the weight restrictions (16).

According to Proposition 5, the NIRS technology $\overline{\mathcal{H}}_{\mathrm{TO}}$ is obtained by changing condition $(17 \mathrm{c})$ to the equality $1^{\top} \lambda=\alpha$, where $\alpha \geq 0$ and $\alpha \leq 1$. It is easy to see that this is equivalent to replacing $(17 \mathrm{c})$ by the " $\leq$ " inequality. Similarly, the NDRS technology $\mathcal{G}_{\mathrm{TO}}$ is obtained by replacing $(17 \mathrm{c})$ by the " $\geq$ " inequality.

Podinovski (2017, Theorem 2) obtains the same technologies $\overline{\mathcal{C}}_{\mathrm{TO}}, \overline{\mathcal{H}}_{\mathrm{TO}}$ and $\mathcal{G}_{\mathrm{TO}}$ in a development requiring an independent proof. Note that these results now simply follow from Corollary 2 and Proposition 5.

\subsection{The hybrid returns-to-scale technology}

The hybrid returns-to-scale (HRS) technology was developed by Podinovski (2004c) for the scenario in which a subset of inputs and outputs are mutually proportional (scalable) but the remaining inputs and outputs are not. Podinovski, Ismail, Bouzdine-Chameeva, and Zhang (2014) report an application of this technology to the assessment of school efficiency in which the number of pupils is assumed proportional to the number of teaching hours. However, the socio-economic factors, including the number of pupils from families of higher socio-economic status and the academic achievements of students are excluded from this proportion. Podinovski and Wan Husain (2017) use a similar approach and utilize the HRS technology in the context of higher education.

Let $X$ and $Y$ be the $m \times n$ and $s \times n$ matrices whose columns are the input and output vectors of the observed DMUs $\left(x_{j}, y_{j}\right), j=1, \ldots, n$, respectively. Let $\tilde{X}$ and $\tilde{Y}$ be the matrices obtained from $X$ and $Y$, respectively, whose rows corresponding to the nonproportional inputs and outputs are changed to zero rows.

The HRS technology $\mathcal{T}_{\text {HRS }}$ is the set of all DMUs $(x, y) \in \mathbb{R}_{+}^{m+s}$ for which there exist vectors $\lambda, \mu, \nu \in \mathbb{R}^{n}$ and slack vectors $s_{x}, s_{y}$ and $s_{\lambda}$ of appropriate dimensions, such that

$$
\begin{aligned}
& X \lambda+\tilde{X} \mu-\tilde{X} \nu+s_{x}=x, \\
& Y \lambda+\tilde{Y} \mu-Y \nu-s_{y}=y, \\
& \lambda-\nu-s_{\lambda}=0 \\
& 1^{\top} \lambda=1, \\
& \lambda, \mu, \nu, s_{x}, s_{y}, s_{\lambda} \geq 0 .
\end{aligned}
$$

It is clear that the above statement is a special case of the general statement (1) of polyhedral technology. In particular, the vector $z$ in (1) corresponds to the combined vector $\left(\lambda, \mu, \nu, s_{x}, s_{y}, s_{\lambda}\right)$ in (18). The parameter conditions (1c) correspond to $n$ homogeneous 
equalities (18c) and the single nonhomogeneous equality (18d). By Corollary 2, to obtain the closed cone extension $\overline{\mathcal{C}}_{\text {HRS }}$ of technology $\mathcal{T}_{\text {HRS }}$, it suffices to remove the nonhomogeneous equality (18d) from the set of conditions (18).

Podinovski (2009) provides an independent derivation of the same technology $\overline{\mathcal{C}}_{\text {HRS }}$, which requires a bespoke mathematical proof. In this paper, we have shown that the same technology $\overline{\mathcal{C}}_{\text {HRS }}$ is immediately obtained by Corollary 2.

It is interesting to note that technology $\overline{\mathcal{C}}_{\text {HRS }}$ may be used not only as a technical tool (reference technology) for evaluating the scale efficiency in technology $\mathcal{T}_{\text {HRS }}$ but also as a suitable model of some actual production processes. An example of this is discussed in Podinovski (2009) in the context of university departments. In this example, the two inputs are teaching and research staff, and the two outputs are students and publications. If we assume full proportionality (scalability) between all inputs and outputs (i.e., CRS) and, additionally, selective proportionality between teaching staff and students, then this production process is correctly modelled by the closed cone technology $\overline{\mathcal{C}}_{\text {HRS }}$. The latter technology is generally larger than the corresponding standard CRS technology of Charnes et al. (1978).

Finally, using Proposition 5, and similar to the example in Section 6.3, the NIRS and NDRS technologies $\overline{\mathcal{H}}_{\text {HRS }}$ and $\mathcal{G}_{\text {HRS }}$ are obtained by replacing the equality (18d) by the " $\leq$ " and " $\geq$ " inequalities, respectively.

\section{The dual perspective}

Assessing the efficiency of any DMU $\left(x_{o}, y_{o}\right)$ in a polyhedral technology $\mathcal{T}$ involves solving a linear program that can be stated in two mutually dual forms referred to as the envelopment and multiplier models.

To be specific, and without loss of generality, consider the evaluation of the output radial efficiency of DMU $\left(x_{o}, y_{o}\right)$ in technology $\mathcal{T}$ stated in the general form (1). This is defined as the inverse of the optimal value of $\theta$ in the following multiplier program:

$$
\begin{aligned}
\max & \theta \\
\text { subject to } & A z=x_{o} \\
& -B z+\theta y_{o}=0 \\
& C z=d \\
& z \geq 0
\end{aligned}
$$

Let $v \in \mathbb{R}^{m}$ and $u \in \mathbb{R}^{s}$ be the vectors of input and output weights defined as dual to constraints $(19 \mathrm{~b})$ and $(19 \mathrm{c})$, respectively. Let $\omega \in \mathbb{R}^{p}$ be the vector of dual variables to the parameter conditions (19d). The multiplier program is the dual to (19):

$$
\begin{aligned}
\min & x_{o}^{\top} v+d^{\top} \omega \\
\text { subject to } & y_{o}^{\top} u=1, \\
& A^{\top} v-B^{\top} u+C^{\top} \omega \geq 0, \\
& u, v, \omega \text { sign free. }
\end{aligned}
$$

For example, for the conventional VRS model defined by (2), $\omega$ is a scalar, and the terms $d^{\top} \omega$ and $C^{\top} \omega$ appearing in program (20) are replaced by $\omega$ and $1^{\top} \omega$, respectively. Also, in the case of VRS (and any other freely disposable technology), the nonnegativity of the vectors of weights $u$ and $v$ follows from the constraints dual to the nonnegative slack vectors $s_{x}$ and $s_{y}$ in program $(2)$. 
Let us show how the multiplier model (20) changes if the technology $\mathcal{T}$ is replaced by its closed cone extension $\overline{\mathcal{C}}$. In this case, by Proposition 3 , an additional variable $\alpha \geq 0$ is introduced in the envelopment program (19), and condition (19c) is replaced by $C z=\alpha d$. The dual multiplier model based on technology $\overline{\mathcal{C}}$ is now stated as follows:

$$
\begin{aligned}
\min & x_{o}^{\top} v \\
\text { subject to } & y_{o}^{\top} u=1, \\
& A^{\top} v-B^{\top} u+C^{\top} \omega \geq 0, \\
& d^{\top} \omega=0, \\
& u, v, \omega \text { sign free. }
\end{aligned}
$$

Comparing programs (20) and (21), note that the latter program has the additional constraint (21d). The absence of the term $d^{\top} \omega$ in the objective function of this program is unimportant. We can formally use the objective function of program (20) in program (21) as well because this term is equal to zero.

It is straightforward to verify that the same modification is required in the case of input radial efficiency, and also for the multiplier models stated in terms of directional distance functions (Chambers et al., 1998). This observation can be formally stated as follows.

Proposition 6. Let $\overline{\mathcal{C}}$ be the closed cone technology generated by polyhedral technology $\mathcal{T}$. Then the multiplier programs based on technology $\overline{\mathcal{C}}$ are obtained from the multiplier programs based on technology $\mathcal{T}$ by incorporating the additional constraint $d^{\top} \omega=0$ in the latter.

As an illustration, consider the closed cone extension $\overline{\mathcal{C}}_{\mathrm{N}}$ of the two-stage network technology $\mathcal{T}_{\mathrm{N}}$ stated in the form (12) in Section 6.1. By Proposition 6, the multiplier models based on technology $\overline{\mathcal{C}}_{\mathrm{N}}$ require the additional constraint

$$
\omega_{\mathrm{I}}+\omega_{\mathrm{II}}=0,
$$

where $\omega_{\text {I }}$ and $\omega_{\text {II }}$ are the dual variables to the first and second-stage normalizing equalities (12e) and (12f), respectively.

Corollary 3. Let the parameter conditions (1c) include a single nonhomogeneous condition, and possibly any other conditions all of which are homogeneous. Let $\omega$ be the dual variable corresponding to this nonhomogeneous condition. Then the multiplier programs based on technology $\overline{\mathcal{C}}$ are obtained from the multiplier programs based on technology $\mathcal{T}$ by incorporating the condition $\omega=0$ in the latter or, equivalently, by removing the variable $\omega$ and all terms with this variable from the program.

An example illustrating Corollary 3 is the conventional CRS technology which is the closed cone technology generated by the VRS technology. The multiplier CRS programs are obtained from the multiplier VRS programs by removing the sign-free variable $\omega$ dual to the convexity constraint (2c).

\section{Conclusion}

The notions of scale efficiency and returns to scale are important in many applications of DEA. According to a well-established methodology, the evaluation of these characteristics in technology $\mathcal{T}$ is based on the use of the reference cone, NIRS and NDRS technologies generated by technology $\mathcal{T}$. These reference technologies are readily available if the underlying technology $\mathcal{T}$ is the VRS technology of Banker et al. (1984) and in some other cases. 
A number of new models of production technologies (often defined axiomatically) have recently been developed in the DEA literature. Most of them are stated in the form of linear equalities and inequalities and are therefore polyhedral technologies. In some cases, the production technology is not stated explicitly but is introduced implicitly by the statement of the multiplier program for the assessment of the input or output radial efficiency of the DMUs, or for the evaluation of the directional distance function of Chambers et al. (1998). For many such technologies their reference technologies have remained unexplored, or have been introduced heuristically, often using unverified analogy with the conventional VRS model.

In this paper, we consider the cone, NIRS and NDRS reference technologies for an arbitrary polyhedral technology $\mathcal{T}$ whose general statement was suggested by Podinovski et al. (2016). Our results show that the required reference technologies are obtained by a modification of the statement of technology $\mathcal{T}$ which is the same for all polyhedral technologies. We also show how the multiplier models should be modified for their use with the reference cone technologies.

Our results should also be of interest for the axiomatic development of polyhedral technologies, to be used as models of the actual production processes, and not only as technical means for the evaluation of scale efficiency. A common approach to this is based on the statement of the production assumptions in the form of axioms. The technology is subsequently defined by the minimum extrapolation principle (Banker et al., 1984) as the intersection of all technologies that satisfy the stated axioms. In many cases, after a particular technology $\mathcal{T}$ has been obtained, a question arises as to how to define the cone (CRS) analogue of $\mathcal{T}$. Answering this question requires stating an additional axiom of scalability of DMUs and formally deriving the required cone technology from the new set of axioms. The results obtained in this paper facilitate this stage of development and show that the statement of the resulting cone technology can easily be obtained by modifying the statement of technology $\mathcal{T}$. 


\section{Appendix A. Proofs}

Proof of Proposition 1. Consider the program obtained from (3) by replacing $\mathcal{T}$ by $\mathcal{C}$ in its constraints:

$$
\begin{aligned}
\sup & \beta / \alpha \\
\text { subject to } & \left(\alpha x_{o}, \beta y_{o}\right) \in \mathcal{C}, \\
& \alpha, \beta>0 .
\end{aligned}
$$

The suprema of programs (3) and (A.1) are equal. Indeed, by (4), any feasible solution of (A.1) is stated as $(\delta \alpha, \delta \beta)$, where $(\alpha, \beta)$ is feasible in (3). Because $(\delta \beta) /(\delta \alpha)=\beta / \alpha$, $\forall \alpha, \delta>0$, the values of the objective function achievable in both programs are the same.

Let $\left(\alpha^{*}, \beta^{*}\right)$ be any feasible solution to program (A.1), for which its objective function is equal to $\beta^{*} / \alpha^{*}$. Then the same value $\beta^{*} / \alpha^{*}$ is achieved at any solution $\left(\delta \alpha^{*}, \delta \beta^{*}\right)$, for any $\delta>0$. In particular, we can take $\delta=1 / \alpha^{*}$, for which we obtain the feasible solution $\left(1, \beta^{*} / \alpha^{*}\right)$. Therefore, the supremum of program (A.1) does not change if we reduce its feasible region to its subset by requiring $\alpha=1$ and remove the now redundant positivity conditions on variables $\alpha$ and $\beta$. This results in program (5).

Let the supremum of (3) be equal to $\beta^{*} / \alpha^{*}$ and attained at some $\left(\alpha^{*} x_{o}, \beta^{*} y_{o}\right) \in \mathcal{T}$. Then the same value is attained in $(5)$ at $\left(x_{o}, \beta^{*} / \alpha^{*} y_{o}\right) \in \mathcal{C}$. Conversely, let the supremum of (5) be equal to $\beta^{*}$ and attained at some $\left(x_{o}, \beta^{*} y_{o}\right) \in \mathcal{C}$. By $(4)$, there exists a $(\tilde{x}, \tilde{y}) \in \mathcal{T}$ and $\delta>0$ such that $\left(x_{o}, \beta^{*} y_{o}\right)=(\delta \tilde{x}, \delta \tilde{y})$. Then $\delta>0$, and the ratio $\beta^{*} / \alpha^{*}$ is attained at $(\tilde{x}, \tilde{y})=\left((1 / \delta) x_{o},\left(\beta^{*} / \delta\right) y_{o}\right) \in \mathcal{T}$.

Proof of Proposition 2. Let $\beta^{*}$ and $\bar{\beta}^{*}$ be the suprema of $\beta$ in programs (7) and (8), respectively. (We allow the case $\beta^{*}=+\infty$ or $\bar{\beta}^{*}=+\infty$.) Because $\mathcal{C} \subseteq \overline{\mathcal{C}}$, we have $\beta^{*} \leq \bar{\beta}^{*}$.

Conversely, consider any $\beta^{\prime} \in\left[1, \bar{\beta}^{*}\right)$. Because $\overline{\mathcal{C}}$ is a convex set and $\left(x_{o}, y_{o}\right) \in \overline{\mathcal{C}}$, by definition of $\bar{\beta}^{*}$, we have $\left(x_{o}-\beta^{\prime} g_{x}, y_{o}+\beta^{\prime} g_{y}\right) \in \overline{\mathcal{C}}$. It suffices to prove that $\beta^{*} \geq \beta^{\prime}$, as this would imply $\beta^{*} \geq \bar{\beta}^{*}$. By Proposition 3 whose proof is independent of this proof, $\left(x_{o}-\beta^{\prime} g_{x}, y_{o}+\beta^{\prime} g_{y}\right)$ satisfies (10) with some $z^{\prime}$ and $\alpha^{\prime}$. Two possibilities arise.

(i) Let $\alpha^{\prime}>0$. Define $(\tilde{x}, \tilde{y})=\left(1 / \alpha^{\prime}\right)\left(x_{o}-\beta^{\prime} g_{x}, y_{o}+\beta^{\prime} g_{y}\right)$. Then $(\tilde{x}, \tilde{y})$ satisfies $(1)$ with $z=1 / \alpha^{\prime}$. Therefore, $(\tilde{x}, \tilde{y}) \in \mathcal{T}$. Because $\left(x_{o}-\beta^{\prime} g_{x}, y_{o}+\beta^{\prime} g_{y}\right)=\left(\alpha^{\prime} \tilde{x}, \alpha^{\prime} \tilde{y}\right)$, by (4), $\left(x_{o}-\beta^{\prime} g_{x}, y_{o}+\beta^{\prime} g_{y}\right) \in \mathcal{C}$. By definition of $\beta^{*}$, we have $\beta^{*} \geq \beta^{\prime}$.

(ii) Let $\alpha^{\prime}=0$. For $k=1,2 \ldots$, define the sequence of units

$$
\begin{aligned}
\left(x^{k}, y^{k}\right) & =\frac{1}{k}\left(x_{o}, y_{o}\right)+\left(1-\frac{1}{k}\right)\left(x_{o}-\beta^{\prime} g_{x}, y_{o}+\beta^{\prime} g_{y}\right) \\
& =\left(x_{o}-\beta_{k} g_{x}, y_{o}+\beta_{k} g_{y}\right)
\end{aligned}
$$

where $\beta_{k}=(1-1 / k) \beta^{\prime}$. Because both $\left(x_{o}, y_{o}\right)$ and $\left(x_{o}-\beta^{\prime} g_{x}, y_{o}+\beta^{\prime} g_{y}\right)$ are in $\overline{\mathcal{C}}$, and $\overline{\mathcal{C}}$ is a convex set, $\left(x^{k}, y^{k}\right) \in \overline{\mathcal{C}}$, for all, $k=1,2, \ldots$

Because $\left(x_{o}, y_{o}\right) \in \mathcal{T}$, it satisfies (1) with some $z=z_{o}$. Therefore, $\left(x_{o}, y_{o}\right)$ satisfies $(10)$ with $z_{o}$ and $\alpha=1$. Then each $\left(x^{k}, y^{k}\right)$ in (A.2) satisfies (10) with $z^{k}=(1 / k) z_{o}+(1-1 / k) z^{\prime}$ and $\alpha^{k}=(1 / k)+(1-1 / k) \alpha^{\prime}$. Note that, for each $k, \alpha^{k}>0$. Then, as proved in Case (i), $\left(x^{k}, y^{k}\right) \in \mathcal{C}$, for each $k$. By definition of $\beta^{*}$, we have $\beta^{*} \geq \beta_{k}$, for all $k$. Because $\beta_{k} \rightarrow \beta^{\prime}$ as $k \rightarrow+\infty$, we have $\beta^{*} \geq \beta^{\prime}$.

Proof of Proposition 3. Let $\mathcal{T}^{*}$ be the technology defined by (10). We need to prove that $\mathcal{T}^{*}=\overline{\mathcal{C}}$, where $\overline{\mathcal{C}}$ is defined by (6). Let us first prove that $\mathcal{C} \subseteq \mathcal{T}^{*}$, where $\mathcal{C}$ is defined by (4). Indeed, let $(x, y) \in \mathcal{C}$. By (4), there exists a $(\tilde{x}, \tilde{y}) \in \mathcal{T}$ and $\delta \geq 0$ such that $(x, y)=(\delta \tilde{x}, \delta \tilde{y})$. Let $(\tilde{x}, \tilde{y})$ satisfy (1) with some $z^{\prime}$. Then $(x, y)$ satisfies (10) with $z=\delta z^{\prime}$ 
and $\alpha=\delta$. Therefore, $(x, y) \in \mathcal{T}^{*}$ and $\mathcal{C} \subseteq \mathcal{T}^{*}$. Taking the closure of both sides, we have $\overline{\mathcal{C}}=\operatorname{cl} \mathcal{C} \subseteq \operatorname{cl} \mathcal{T}^{*}=\mathcal{T}^{*}$. (The last equality follows from the fact that $\mathcal{T}^{*}$ is a polyhedral and therefore a closed technology.)

It remains to be proved that $\mathcal{T}^{*} \subseteq \overline{\mathcal{C}}$. Consider any $\left(x^{\prime}, y^{\prime}\right) \in \mathcal{T}^{*}$. Then $\left(x^{\prime}, y^{\prime}\right)$ satisfies (10) with some $z^{\prime}$ and $\alpha^{\prime}$. Two possibilities arise.

(i) Let $\alpha^{\prime}>0$. Define $(\tilde{x}, \tilde{y})=\left(1 / \alpha^{\prime}\right)\left(x^{\prime}, y^{\prime}\right)$. Then $(\tilde{x}, \tilde{y})$ satisfies (1) with $z=1 / \alpha^{\prime}$. Therefore, $(\tilde{x}, \tilde{y}) \in \mathcal{T}$. Because $\left(x^{\prime}, y^{\prime}\right)=\left(\alpha^{\prime} \tilde{x}, \alpha^{\prime} \tilde{y}\right)$, by $(4)$ and $(6),\left(x^{\prime}, y^{\prime}\right) \in \mathcal{C} \subseteq \overline{\mathcal{C}}$.

(ii) Let $\alpha^{\prime}=0$. Let us prove that $\left(x^{\prime}, y^{\prime}\right)$ is a limit point of $\mathcal{C}$ and is therefore in $\overline{\mathcal{C}}$. Consider any $\left(x^{*}, y^{*}\right) \in \mathcal{T}$ which satisfies (1) with some $z^{*}$. Therefore, $\left(x^{*}, y^{*}\right)$ satisfies (10) with $z=z^{*}$ and $\alpha=1$. For $k=1,2 \ldots$, define the sequence of units

$$
\left(x^{k}, y^{k}\right)=\frac{1}{k}\left(x^{*}, y^{*}\right)+\left(1-\frac{1}{k}\right)\left(x^{\prime}, y^{\prime}\right) .
$$

Each $\left(x^{k}, y^{k}\right)$ satisfies (10) with $z^{k}=(1 / k) z^{*}+(1-1 / k) z^{\prime}$ and $\alpha^{k}=(1 / k)+(1-1 / k) \alpha^{\prime}$. Therefore, $\left(x^{k}, y^{k}\right) \in \mathcal{T}^{*}$, for all $k=1,2, \ldots$ For each $k, \alpha^{k}>0$ and, as proved in Case (i), $\left(x^{k}, y^{k}\right) \in \overline{\mathcal{C}}$. The limit of the sequence of units $\left(x^{k}, y^{k}\right)$ is $\left(x^{\prime}, y^{\prime}\right)$. Because $\overline{\mathcal{C}}$ is a closed set, $\left(x^{\prime}, y^{\prime}\right) \in \overline{\mathcal{C}}$.

Proof of Corollary 2. Let $t^{\prime} \in\{1, \ldots, p\}$ be the single nonhomogeneous condition in (1c). Denote $\mathcal{T}^{*}$ the technology defined by conditions (1) from which the condition $t^{\prime}$ in the group (1c) is removed. We need to prove that $\overline{\mathcal{C}}=\mathcal{T}^{*}$. By Proposition 3 , any $(x, y) \in \overline{\mathcal{C}}$ satisfies conditions (10) with some $z^{\prime}$ and $\alpha^{\prime}$. Then $(x, y)$ satisfies $(1)$, with the nonhomogeneous condition $t^{\prime}$ removed, with the same $z^{\prime}$. Therefore, $(x, y) \in \mathcal{T}^{*}$ and $\overline{\mathcal{C}} \subseteq \mathcal{T}^{*}$.

Conversely, let $(x, y) \in \mathcal{T}^{*}$ satisfy conditions (1), from which the nonhomogeneous condition $t^{\prime}$ with the right-hand side $d_{t^{\prime}} \neq 0$ is removed, with some $z^{\prime}$. Define $\alpha^{\prime}=D z^{\prime} / d_{t^{\prime}}$. Then $(x, y)$ satisfies (10) with $z^{\prime}$ and $\alpha^{\prime}$. Therefore, $(x, y) \in \overline{\mathcal{C}}$ and $\mathcal{T}^{*} \subseteq \overline{\mathcal{C}}$.

Proof of Proposition 4. According to Proposition 5, $\mathcal{G}$ is a polyhedral set. Therefore, $\mathcal{G}$ is a closed set.

Proof of Proposition 5. The proof of this result is similar to the proof of Proposition 3 and is only outlined. Consider technology $\overline{\mathcal{H}}$. Denote $\mathcal{T}^{*}$ the technology defined by (10) and the additional condition $\alpha \leq 1$. It is straightforward to show that $\overline{\mathcal{H}} \subseteq \mathcal{T}^{*}$. The inverse embedding is proved by considering Cases (i) and (ii) similar to those in the proof of Proposition 3. The case of technology $\mathcal{G}$ is similar. For this technology, $\alpha \geq 1$, and only Case (1) is possible. 


\section{References}

Allen, R., Athanassopoulos, A., Dyson, R. G., \& Thanassoulis, E. (1997). Weights restrictions and value judgements in data envelopment analysis: Evolution, development and future directions. Annals of Operations Research, 73, 13-34.

Banker, R. D. (1984). Estimating most productive scale size using data envelopment analysis. European Journal of Operational Research, 17(1), 35-44.

Banker, R. D., Charnes, A., \& Cooper, W. W. (1984). Some models for estimating technical and scale efficiencies in data envelopment analysis. Management Science, 30(9), 1078-1092.

Briec, W., Kerstens, K., Leleu, H., \& Vanden Eeckaut, P. (2000). Returns to scale on nonparametric deterministic technologies: Simplifying goodness-of-fit methods using operations on technologies. Journal of Productivity Analysis, 14(3), 267-274.

Cesaroni, G., \& Giovannola, D. (2015) Average-cost efficiency and optimal scale sizes in non-parametric analysis. European Journal of Operational Research, 242(1), 121-133.

Cesaroni, G., Kerstens, K., \& Van de Woestyne, I. (2017) Global and local scale characteristics in convex and nonconvex nonparametric technologies: A first empirical exploration. European Journal of Operational Research, 259(2), 576-586.

Chambers, R. G., Chung, Y., \& Färe, R. (1998). Profit, directional distance functions, and Nerlovian efficiency. Journal of Optimization Theory and Applications, 98(2), 351-364.

Charnes, A., Cooper, W. W., \& Rhodes, E. (1978). Measuring the efficiency of decision making units. European Journal of Operational Research, 2(6), 429-444.

Cherchye, L., De Rock, B., Dierynck, B., Roodhooft, F., \& Sabbe, J. (2013). Opening the "black box" of efficiency measurement: Input allocation in multioutput settings. Operations Research, 61 (5), 1148-1165.

Cooper, W. W., Pastor, J. T., Borras, F., Aparicio, J., \& Pastor, D. (2011). BAM: A bounded adjusted measure of efficiency for use with bounded additive models Journal of Productivity Analysis, 35 (2), 85-94.

Deprins, D., Simar, L., \& Tulkens, H. (1984). Measuring labor-efficiency in post offices. In M. Marchand, P. Pestieau, \& H. Tulkens (Eds.), The performance of public enterprises: concepts and measurements (pp. 243-267). Amsterdam: North-Holland.

Dyson, R. D., \& Thanassoulis, E. (1988). Reducing weight flexibility in data envelopment analysis. Journal of the Operational Research Society, 39(6), 563-576.

Färe, R., \& Grosskopf, S. (1985). A nonparametric cost approach to scale efficiency. Scandinavian Journal of Economics, $87(4), 594-604$.

Färe, R., Grosskopf, S., \& Lovell, C. A. K. (1983). The structure of technical efficiency. Scandinavian Journal of Economics, 85(2), 181-190.

Färe, R., Grosskopf, S., \& Lovell, C. A. K. (1985). The measurement of efficiency of production. Boston: Kluwer Academic Publishers.

Färe, R., Grosskopf, S., \& Whittaker, G. (2007). Network DEA. In J. Zhu, \& W. D. Cook (Eds.), Modeling data irregularities and structural complexities in data envelopment analysis (pp. 209-240). New York: Springer Science + Busines Media.

Kao, C. (2014). Network data envelopment analysis: A review. European Journal of Operational Research, $239(1), 1-16$.

Kao, C. (2017). Network data envelopment analysis: Foundations and Extensions. New York: Springer Science + Busines Media.

Kerstens, K., \& Vanden Eeckaut, P. (1999). Estimating returns to scale using non-parametric deterministic technologies: A new method based on goodness-of-fit. European Journal of Operational Research, 113(1), 206-214.

Kuosmanen, T. (2005). Weak disposability in nonparametric productivity analysis with undesirable outputs. American Journal of Agricultural Economics, 87(4), 1077-1082.

Liang, L., Cook, W. D., Zhu, J. (2008). DEA models for two-stage processes: Game approach and efficiency decomposition. Naval Research Logistics, 55(7), 643-653.

Mehdiloozad, M., \& Podinovski, V. V. (2018). Nonparametric production technologies with weakly disposable inputs. European Journal of Operational Research, 266(1), 247-258.

Pastor, J. T., Aparicio, J., Alcaraz, J., Vidal, F., \& Pastor, D. (2015). An enhanced BAM for unbounded or partially bounded CRS additive models. Omega, 56, 16-24.

Podinovski, V. V. (2004a). Efficiency and returns to scale on the "no free lunch assumption only. Journal of Productivity Analysis, 22(3), 227-257.

Podinovski, V. V. (2004b). Local and global returns to scale in performance measurement. Journal of the Operational Research Society, 55(2), 170-178. 
Podinovski, V. V. (2004c). Bridging the gap between the constant and variable returns-to-scale models: Selective proportionality in data envelopment analysis. Journal of the Operational Research Society, 55(3), 265-276.

Podinovski, V. V. (2004d). Production trade-offs and weight restrictions in data envelopment analysis. Journal of the Operational Research Society, 55(12), 1311-1322.

Podinovski, V. V. (2009). Production technologies based on combined proportionality assumptions. Journal of Productivity Analysis, 32(1), 21-26.

Podinovski, V. V. (2015). DEA models with production trade-offs and weight restrictions. In J. Zhu (Ed.), Data envelopment analysis: A handbook of models and methods (pp. 105-144). New York: Springer Science + Busines Media.

Podinovski, V. V. (2017). Returns to scale in convex production technologies. European Journal of Operational Research, 258(3), 970-982.

Podinovski, V. V., \& Bouzdine-Chameeva, T. (2013). Weight restrictions and free production in data envelopment analysis. Operations Research, 61(2), 426-437.

Podinovski, V. V., Chambers, R. G., Atici, K. B., \& Deineko, I. D. (2016). Marginal values and returns to scale for nonparametric production frontiers. Operations Research, 64 (1), 236-250.

Podinovski, V. V., Ismail, I., Bouzdine-Chameeva, T., \& Zhang, W. (2014). Combining the assumptions of variable and constant returns to scale in the efficiency evaluation of secondary schools. European Journal of Operational Research, 239(2), 504-513.

Podinovski, V. V., Olesen, O. B., \& Sarrico, S. C. (2018). Nonparametric production technologies with multiple component processes. Operations Research, 66(1), 282-300.

Podinovski, V. V., \& Wan Husain, W. R. (2017). The hybrid returns-to-scale model and its extension by production trade-offs: An application to the efficiency assessment of public universities in Malaysia. Annals of Operations Research, 250(1), 65-84.

Rockafellar, R. T. (1970). Convex analysis. Princeton, NJ: Princeton University Press.

Rockafellar, R. T., \& Wets, R. J.-B. (1998). Variational analysis. Berlin: Springer-Verlag.

Sahoo, B. K., Zhu, J., Tone, K., \& Klemen, B. M. (2014). Decomposing technical efficiency and scale elasticity in two-stage network DEA. European Journal of Operational Research, 233(3), 584-594.

Soleimani-damaneh, M., Jahanshahloo, G. R., \& Reshadi, M. (2006). On the estimation of returns-to-scale in FDH models. European Journal of Operational Research, 174(2), 1055-1059. 